\title{
Aerosol Performance and Long-Term Stability of Surfactant-Associated Liposomal Ciprofloxacin Formulations with Modified Encapsulation and Release Properties
}

\author{
David Cipolla, ${ }^{1,2,3}$ Huiying Wu, ${ }^{2}$ Igor Gonda, ${ }^{2}$ and Hak-Kim Chan ${ }^{1}$
}

Received 29 December 2013; accepted 14 May 2014; published online 3 June 2014

\begin{abstract}
Previously, we showed that the encapsulation and release properties of a liposomal ciprofloxacin formulation could be modified post manufacture, by addition of surfactant in concert with osmotic swelling of the liposomes. This strategy may provide more flexibility and convenience than the alternative of manufacturing multiple batches of liposomes differing in composition to cover a wide range of release profiles. The goal of this study was to develop a surfactant-associated liposomal ciprofloxacin (CFI) formulation possessing good long-term stability which could be delivered as an inhaled aerosol. Preparations of $12.5 \mathrm{mg} / \mathrm{ml} \mathrm{CFI} \mathrm{containing} 0.4 \%$ polysorbate 20 were formulated between $\mathrm{pH} 4.7$ and 5.5 . These formulations, before and after mesh nebulization, and after refrigerated storage for up to 2 years, were characterized in terms of liposome structure by cryogenic transmission electron microscopy (cryo-TEM) imaging, vesicle size by dynamic light scattering, $\mathrm{pH}$, drug encapsulation by centrifugation-filtration, and in vitro release (IVR) performance. Within the narrower $\mathrm{pH}$ range of 4.9 to 5.2, these formulations retained their physicochemical stability after 2-year refrigerated storage, were robust to mesh nebulization, and formed respirable aerosols with a volume mean diameter (VMD) of $3.7 \mu \mathrm{m}$ and a geometric standard deviation (GSD) of 1.7. This study demonstrates that it may be possible to provide a range of release profiles by simple addition of surfactant to a liposomal formulation post manufacture, and that these formulations may retain their physicochemical properties after long-term refrigerated storage and following aerosolization by mesh nebulizer.
\end{abstract}

KEY WORDS: ciprofloxacin; drug delivery; liposome; nebulized aerosol; surfactant.

\section{INTRODUCTION}

Liposomes represent a versatile formulation and delivery technology that can be used to modulate a drug's pharmacokinetic profile with the potential to improve both the safety and efficacy of treatment $(1,2)$. Liposomal formulations are now well-accepted with more than ten liposomal pharmaceutical products approved to treat a variety of indications as diverse as fungal infection, ovarian cancer, myeloma, macular degeneration, and leukemia $(2,3)$. The physicochemical and release properties can be designed into the liposomal formulation through appropriate selection of a number of variables including the lipid and sterol composition, the ratio of sterol to lipid, the addition of surface-modifying groups (e.g., polyethylene glycol), the size and lamellarity of the liposomes, the

\footnotetext{
$\overline{{ }^{1} \text { Advanced Drug Delivery Group, Faculty of Pharmacy, The University }}$ of Sydney, Pharmacy Building A15, Sydney, NSW 2006, Australia.

${ }^{2}$ Aradigm Corporation, 3929 Point Eden Way, Hayward, California 94545, USA.

${ }^{3}$ To whom correspondence should be addressed. (e-mail: cipollad@aradigm.com)
}

method of drug loading, the state of the drug inside the liposomes, and the ratio of drug to lipid $(1,4,5)$.

Generally, once a liposome formulation has been manufactured, its drug release properties are not typically modifiable. Thus, in order to provide personalized therapy to individuals, who may optimally respond to distinct drug release profiles, multiple batches of liposomes would have to be manufactured possessing different release kinetics. Because of the complexity and expense of this approach, from both a manufacturing and regulatory perspective, none of the currently marketed liposomal products come in multiple formats each with its own unique release behavior. A simple and flexible approach which allows modification of the drug release rate of a single liposomal formulation post manufacture, could address this issue.

In a previous study, we demonstrated that the encapsulation and drug release properties of a liposomal ciprofloxacin formulation could be altered by addition of surfactant in combination with osmotic swelling of the liposome (6). In absence of osmotic swelling, the surfactant caused only a small change in the drug encapsulation state ( $6 \%$ loss of encapsulated drug for $12.5 \mathrm{mg} / \mathrm{mL}$ CFI mixed with $0.4 \%$ polysorbate 20 ) and there was no change in the IVR profile (6). In contrast, for 
$12.5 \mathrm{mg} / \mathrm{mL}$ CFI mixed with $0.4 \%$ polysorbate 20 under hypotonic conditions, the drug encapsulation state decreased by $\sim 30 \%$ and this formulation also had a much faster IVR profile than the unmodified formulation (6). In the present study, we were interested whether surfactant-associated liposome formulations could be designed to retain their physicochemical properties under long-term refrigerated storage and following aerosol delivery using nebulizers.

Currently, there are no liposomal formulations approved for delivery by the inhalation route. However, the potential for liposomal formulations of drugs to be used to treat lung disease has been intensively investigated, both in terms of understanding the in vitro requirements and the medical opportunities $(2,3,7,8)$. A marketed liposomal formulation of amphotericin B has been used off-label in lung transplant patients as a prophylaxis to prevent fungal infections and was found to be better tolerated than formulations of free amphotericin B (3). Repeated inhalation of amphotericin B liposomes also did not appear to alter the composition of the lipid and surfactant pool in the lungs, nor were there any changes in lung function, suggesting an acceptable safety profile (9). Two inhaled liposomal antibiotic formulations are now in late stages of clinical development to treat lung infection $(2,10)$.

Inhaled ARIKACE®, liposomal amikacin (Insmed, Monmouth Junction, NJ), in cystic fibrosis (CF) patients, was found to reduce Pseudomonas aeruginosa (PA) colonization and improve lung function at the end of each of the six 28-day treatment cycles (11). In a Phase 3 trial in CF patients designed to support regulatory filing in Europe and Canada, once-daily ARIKACE met the primary endpoint of non-inferiority to inhaled tobramycin solution dosed twice-daily (12). Inhaled ARIKACE is also being evaluated in a Phase 2 trial in 90 patients with nontuberculous mycobacteria (NTM) lung infections and enrollment was recently completed; the primary efficacy data of change in mycobacteria density are expected to be reviewed and released to the FDA before the end of Q1, 2014. Pulmaquin ${ }^{\circledR}$ (Aradigm Corporation, Hayward, CA), a dual release formulation of liposomal ciprofloxacin and unencapsulated ciprofloxacin with an in vivo half-life of $\sim 10 \mathrm{~h}$, achieved the primary and a secondary measure of efficacy in a Phase 2 trial in non-CF bronchiectasis and has cleared a US IND to enter Phase 3 trials in that indication (13).

In addition to meeting all specification criteria typical for pharmaceutical products, including those used to verify strength, quality, purity, and potency of the product, liposomal formulations intended for inhalation should also be robust to the aerosolization process (2). Most inhaled liposomal formulations have been developed for use with nebulizers but the nebulization process often compromises the state of drug encapsulation or modifies the vesicle size distribution (2). The presence of surfactant in the liposome formulation may serve to exacerbate this tendency for disruption by nebulization. In fact, a liposome formulation containing polysorbate 80 to increase its deformability was found to be unstable to jet, ultrasonic, and vibrating mesh nebulization with release of up to $98 \%$ of the encapsulated salbutamol sulfate drug (14). Thus, it might be expected that surfactant-modified liposome formulations, with altered encapsulation and release profiles, may be inherently unstable to the nebulization process. Thus, the selected formulations were placed on long-term stability and also evaluated for their stability in response to mesh nebulization. Jet nebulizers were also evaluated but rejected because the presence of surfactant caused excessive foaming in those devices. The goal of this study was to identify surfactant-associated liposome formulations that retained their physicochemical properties on stability and following mesh nebulization. This research is consistent with the overarching mission of personalized medicine by developing a simple methodology, addition of surfactant, to modify the release properties of an inhaled liposomal formulation.

\section{MATERIALS AND METHODS}

\section{Materials}

Lipoquin, liposomal ciprofloxacin (CFI), $50 \mathrm{mg} / \mathrm{ml}$ in a histidine-buffered aqueous formulation at $\mathrm{pH} 6.0$ and free ciprofloxacin (FCI), $20 \mathrm{mg} / \mathrm{ml}$, in an acetate-buffered aqueous formulation at $\mathrm{pH} 3.3$ were from Aradigm Corporation (Hayward, CA). Nanosep centrifugal filtration devices, $10 \mathrm{~K}$ and $30 \mathrm{~K}$ molecular weight, were obtained from Pall Corporation (Ann Arbor, MI). Deionized water was used for all studies. Polysorbate 20 was purchased from VWR Int. (West Chester, NJ) while HEPES, free acid was purchased from Avantor (Center Valley, PA). Sodium chloride and sodium acetate were obtained from Amresco (Solon, $\mathrm{OH}$ ) and Sigma-Aldrich (St. Louis, MO), respectively. HPLC grade methanol was purchased from Fisher Scientific (Fair Lawn, NJ) and Triethylamine (TEA) and HPLC grade methanol were obtained from HyClone (Logan, Utah) and JT Baker (Center Valley, Pennsylvania), respectively.

\section{Preparation of Liposomal Ciprofloxacin}

Lipoquin is an aqueous dispersion of unilamellar liposomes of $\sim 80 \mathrm{~nm}$ containing hydrogenated soy phosphatidylcholine (HSPC) and cholesterol. The preparation of Lipoquin, liposomal ciprofloxacin (CFI), has been reported previously $(15,16)$. Briefly, multilamellar liposomes are extruded through membranes to produce unilamellar liposomes which are then actively loaded with ciprofloxacin $(17,18)$. Any unencapsulated ciprofloxacin is removed by diafiltration resulting in $>99 \%$ encapsulated ciprofloxacin at a target concentration of $50 \mathrm{mg} / \mathrm{ml}$ ciprofloxacin. Pulmaquin, dual release ciprofloxacin for inhalation (DRCFI), is an equivolume mixture of FCI and CFI resulting in $\sim 70 \%$ encapsulated and $\sim 30 \%$ free ciprofloxacin.

\section{Preparation and Selection of CFI Formulations for Long- Term Stability}

CFI was diluted from $50 \mathrm{mg} / \mathrm{ml}$ to $12.5 \mathrm{mg} / \mathrm{ml}$ with water, sodium acetate buffer $(25 \mathrm{mM}$ sodium acetate, $145 \mathrm{mM} \mathrm{NaCl}$, $\mathrm{pH} 4.0$ ), and polysorbate 20 , to achieve a final polysorbate 20 concentration of $0.4 \%(w / v)$. The target $\mathrm{pH}$ was 5.5 (for the unadjusted formulation), and 5.2, 4.9, and 4.7 for the three $\mathrm{pH}$ adjusted formulations, by varying the proportion of water or sodium acetate buffer that was added. These four formulations containing $12.5 \mathrm{mg} / \mathrm{ml} \mathrm{CFI,} 0.4 \%$ polysorbate 20 , but 
differing in $\mathrm{pH}$ and osmolarity, were stored at refrigerated conditions for evaluation of long-term stability. Fifty milliliters of each formulation was prepared and $\sim 6 \mathrm{ml}$ aliquots were pipetted into eight $10 \mathrm{ml}$ glass vials and stored at $2-8^{\circ} \mathrm{C}$ until use. At various time points, including initial, 1 month, 2.5 months, 6 months, 1 year, and 2 years, vials were removed for analysis of their appearance, $\mathrm{pH}$, vesicle size distribution, and drug encapsulation. The IVR profile was determined at the initial, 6-month and 1-year time points. The aerosol properties were determined at the initial, 1-month, and 6-month time points.

\section{Aerosol Characterization}

The aerosol particle size distribution of the $12.5 \mathrm{mg} / \mathrm{ml}$ CFI formulation containing $0.4 \%$ polysorbate $20, \mathrm{pH} 5.5$ was determined using laser diffraction (HELOS/BF, Sympatec $\mathrm{GmbH}$, Clausthal-Zellerfeld, Germany). The aerosol output was drawn at $12.5 \mathrm{~L} / \mathrm{min}$ through a flow-through cell in front of the optical lens and twenty time slices of $500 \mu$ s each were analyzed. WINDOX 5 software was used assuming a shape factor of 1.00 , density of $1.019 \mathrm{~g} / \mathrm{cm}^{3}$, and Mie analysis mode to calculate a volume mean diameter (VMD) and geometric standard deviation (GSD).

At the initial time point, $5 \mathrm{ml}$ aliquots of the control CFI formulation and the $\mathrm{pH} 5.5,4.9$, and 4.7 CFI formulations containing $0.4 \%$ polysorbate 20 were loaded into the Pari eFlow ${ }^{\circledR}$ rapid nebulizer reservoir (Pari Pharma, Starnberg, Germany) with a $4-\mu \mathrm{m}$ mesh, the nebulizer was turned on, and the aerosol output was collected in an SKC BioSampler (USA) containing $5 \mathrm{ml}$ of $25 \mathrm{mM}$ acetate buffer, $\mathrm{pH}$ 4.0. The nebulization time and mass of liposomal ciprofloxacin in the nebulizer residual and collected aerosol compartments were determined in each of the duplicate experiments. The mean vesicle size and state of encapsulation were determined before nebulization and after nebulization for the formulation recovered in the nebulizer reservoir and the collected aerosol. In subsequent characterization experiments at the 1-month and 6-month time points, the aerosol evaluation was performed on the formulation which accumulated in the device mouthpiece, rather than drawing the aerosol into the SKC Biosampler, as it was suspected that the collection in the Biosampler could modify the vesicle size. The sample from the mouthpiece represented impaction of droplets that had passed through the mesh and were exposed to the aerosolization process. For the 1-month and 6-month time points, both the vesicle size and encapsulation state were additionally measured. The IVR profiles of the aerosolized samples were characterized at the 6-month time point only.

\section{pH}

A Beckman Phi690 pH Meter (Fullerton, CA) with a Beckman Coulter combination pH electrode, Model 511275 (Fullerton, CA), was calibrated using Fisher (Fairlawn, NJ) $\mathrm{pH} 4.00$ and 6.00 buffer standards and then used to measure the $\mathrm{pH}$ of the experimental samples.

\section{Vesicle Size}

To measure the liposome size distribution of each experimental CFI sample, a Submicron Particle Sizer Autodilute
Model 370 (Nicomp, USA) was used. Each CFI sample was diluted with isotonic saline to a concentration of $\sim 2 \mathrm{mg} / \mathrm{ml}$ liposomes and $0.5 \mathrm{ml}$ was transferred to a disposable culture tube (Kimble Glass Inc., USA). The mean and standard deviation (SD) of the vesicle size distribution were recorded using the following instrument parameters: temperature, $23^{\circ} \mathrm{C}$; viscosity, 0.933; refractive index, 1.333; intensity set point, $300 \mathrm{KHz}$; channel width, $10 \mu \mathrm{s}$; scattering angle, $90^{\circ}$; run time, 5 min; mode, vesicle; and Gaussian distribution.

\section{Drug Encapsulation}

The percent drug encapsulation was determined by separating the free drug from the encapsulated drug using Nanosep centrifugation devices. Briefly, each sample was diluted tenfold into acetate buffer $(50 \mathrm{mM}$ sodium acetate, $145 \mathrm{mM} \mathrm{NaCl}, \mathrm{pH} 4.0$ ) and $400 \mu \mathrm{L}$ was transferred to the centrifuge device and centrifuged at $10,000 \mathrm{rpm}(8,100 \times \mathrm{g})$. The filtrate containing the free ciprofloxacin component was analyzed by HPLC to measure ciprofloxacin content. The percent encapsulation of each sample was calculated by dividing the free drug component by the total drug content and multiplying by 100 to convert to a percentage.

\section{Cryogenic Transition Electron Microscopy (cryo-TEM)}

Cryo-TEM analysis was performed using a JEOL 2100 (Tokyo, Japan) instrument operated at $200 \mathrm{kV}$ to image the liposome vesicles. Each sample was diluted with water to a liposome concentration of $\sim 10 \mathrm{mg} / \mathrm{ml}$ lipids and $3 \mu \mathrm{L}$ was transferred to a glow discharge Quantifoil carbon grid (Jena, Germany) in a chamber equilibrated at $22^{\circ} \mathrm{C}$ and $100 \% \mathrm{RH}$. Grids were blotted once with filter paper, at a blotting angle of $2 \mathrm{~mm}$ for $2 \mathrm{~s}$, and vitrified by plunging into liquid ethane using a Vitrobot (F.E.I., Eindhoven, Netherlands). To ensure sample integrity prior to cryo-TEM analysis, the vitrified samples remained immersed in liquid nitrogen.

\section{In Vitro Release (IVR)}

The IVR assay measures the release of encapsulated ciprofloxacin when incubated at $37^{\circ} \mathrm{C}$ in $50 \%$ bovine serum (16). Briefly, each experimental sample was diluted to $50 \mu \mathrm{g} /$ $\mathrm{ml}$ ciprofloxacin in HEPES Buffered Saline (HBS, $20 \mathrm{mM}$ HEPES; $145 \mathrm{mM} \mathrm{NaCl}, \mathrm{pH}$ 7.4) and mixed one-to-one with chilled $\left(2-8^{\circ} \mathrm{C}\right)$ bovine serum and placed in a shaking water bath (Techne, TSBS40 (Staffordshire, UK)) at $37^{\circ} \mathrm{C}$ and $150 \mathrm{rpm}$. Duplicate samples were removed periodically; e.g., 30, 60, 120, and $240 \mathrm{~min}$, diluted 1:1 with chilled HBS buffer, and placed in an ice water bath to terminate any further release of encapsulated drug from the liposomes. The released ciprofloxacin was separated from the liposome-encapsulated ciprofloxacin using the Nanosep centrifugal devices and quantified by HPLC as described previously (16). The percent release at each time point was determined by dividing the free drug by the total drug and multiplying by 100 to convert to a percentage. In order to compare the initial release rates of different formulations with different percentages of encapsulated drug, a normalized initial rate of release was calculated. This was done by subtracting the percentage of drug released initially from that released at the $30 \mathrm{~min}$ time point; i.e., 
$\left(\mathrm{T}_{30 \min }-\mathrm{T}_{0 \min }\right)$. This initial release value was then divided by the total available range for release $\left(\mathrm{T}_{240 \min }-\mathrm{T}_{0 \mathrm{~min}}\right)$ and converted to a percentage: $100 \times\left(\mathrm{T}_{30 \min }-\mathrm{T}_{0 \min }\right) /\left(\mathrm{T}_{240 \min }-\mathrm{T}_{0 \min }\right)$.

\section{High Performance Liquid Chromatography (HPLC)}

An HPLC method was used to quantify the amount of ciprofloxacin in each sample as described previously (19). Briefly, this was achieved using a Nucleosil C-18 column (5 $\mu \mathrm{m}, 4.6 \times 150 \mathrm{~mm}$, Canadian Life Science, CA) and a Nucleosil C-18 guard column $(4 \times 3.0 \mathrm{~mm}$, Phenomenex, USA) both at $35^{\circ} \mathrm{C}$. The mobile phase was an 83:17 (v/v) mixture of $0.5 \%$ TEA in water, pH 3.0 and $100 \%$ methanol. The isocratic elution was conducted at a flow rate was $0.9 \mathrm{ml} /$ min. A wavelength of $277 \mathrm{~nm}$ was used to quantify ciprofloxacin content. Ciprofloxacin concentrations were expressed in terms of ciprofloxacin hydrochloride.

\section{RESULTS}

\section{Selection of CFI Formulations for Long-Term Stability}

Dilution of $50 \mathrm{mg} / \mathrm{mL}$ CFI to $12.5 \mathrm{mg} / \mathrm{mL}$ with polysorbate 20 and water, instead of histidine buffer, to produce a hypotonic environment surrounding the liposomes, resulted in much greater release of the encapsulated ciprofloxacin (Fig. 1). Polysorbate 20 when added to CFI in a hypotonic environment at a concentration between 0.4 and $1.6 \%$, caused $\sim 30$ to $40 \%$ of the encapsulated ciprofloxacin to be released from the liposomes, versus only $\sim 6$ to $8 \%$ release when the surfactant was added to CFI under isotonic conditions (Fig. 1). The combination of osmotic swelling of the liposomes, in concert with surfactant, allowed for more of the encapsulated drug to be released (6). In addition, the $12.5 \mathrm{mg} / \mathrm{mL}$ CFI formulation containing $0.4 \%$ polysorbate 20 when prepared in a hypotonic environment also possessed a faster release rate in the IVR assay, whereas lower concentrations of polysorbate 20 did not significantly alter the release rate (Fig. 2a). The CFI formulation containing $0.4 \%$ polysorbate 20 was compared to DRCFI, which is a $\sim 70: 30$ mixture of CFI and unencapsulated ciprofloxacin, in the IVR assay and both possessed a comparable "burst" value of $\sim 30 \%$, but the surfactant-modified CFI formulation had a faster release profile than DRCFI (Fig. 2a). After 2 to $4 \mathrm{~h}$ incubation in the IVR assay, all formulations released nearly $100 \%$ of the encapsulated drug (Fig. 2a).

Based on these results, the $12.5 \mathrm{mg} / \mathrm{mL}$ CFI formulation containing $0.4 \%$ polysorbate 20 added under hypotonic conditions was selected for further studies for its potential as an inhaled product. However, because ciprofloxacin is poorly soluble near neutral $\mathrm{pH}$, this unbuffered formulation was not expected to possess good long-term stability. In fact, rodshaped crystals of ciprofloxacin were observed external to the liposomes under light microscope within $48 \mathrm{~h}$ of addition of the surfactant when the $\mathrm{pH}$ was not adjusted from $\mathrm{pH}$ 5.5. In order to develop a formulation with acceptable long-term stability, the $50 \mathrm{mg} / \mathrm{ml} \mathrm{CFI} \mathrm{formulation} \mathrm{was} \mathrm{diluted} \mathrm{with} \mathrm{poly-}$ sorbate 20 , sodium acetate buffer, and water to produce $12.5 \mathrm{mg} / \mathrm{mL}$ CFI formulations containing $0.4 \%$ polysorbate 20 and $\mathrm{pH}$ values of 5.2, 4.9, and 4.7. These formulations were stored refrigerated for periodic evaluation of appearance, vesicle size distribution, ciprofloxacin encapsulation, $\mathrm{pH}$, and IVR profile.

\section{Long-Term Stability}

Appearance, Vesicle Size, and $p H$

The visual appearance of the $\mathrm{pH} 4.7$ and 4.9 CFI formulations containing $0.4 \%$ polysorbate 20 remained particle-free throughout the 2-year study while a small number of fine particles were observed in the $\mathrm{pH} 5.2$ formulation at the 2.5, 6,12 , and 24-month time points. Presumably, the small particles were fine crystals of ciprofloxacin external to the liposomes. The mean liposome vesicle size ranged between 89 and $100 \mathrm{~nm}$ across all four formulations through the 2-year time point (Table I). There was no meaningful change in vesicle size for any of the three formulations during the 1-year study. The $\mathrm{pH}$ was relatively unchanged for the $\mathrm{pH} 4.7,4.9$, and 5.2 CFI formulations containing $0.4 \%$ polysorbate 20 through 2 years (Table II).

\section{Encapsulation}

The encapsulation of the $50 \mathrm{mg} / \mathrm{mL}$ CFI control sample remained at $\sim 99 \%$ over the $24-$ month period (Table III). The $12.5 \mathrm{mg} / \mathrm{ml} \mathrm{CFI}$ formulation at $\mathrm{pH} 5.5$ containing $0.4 \%$ polysorbate 20 had between 70 and $75 \%$ drug encapsulation initially, but these samples were not monitored on stability due to crystallization of the released ciprofloxacin. For the $\mathrm{pH}$ adjusted formulations, the initial percent encapsulation decreased from $99 \%$ to $\sim 80, \sim 85$, and $\sim 92 \%$ when diluted with sodium acetate buffer and water to lower the $\mathrm{pH}$ to 5.2, 4.9, and 4.7, respectively (Table III). The more acetate buffer that was used to reduce the $\mathrm{pH}$ correlated with higher starting encapsulation values, consistent with the mechanism that

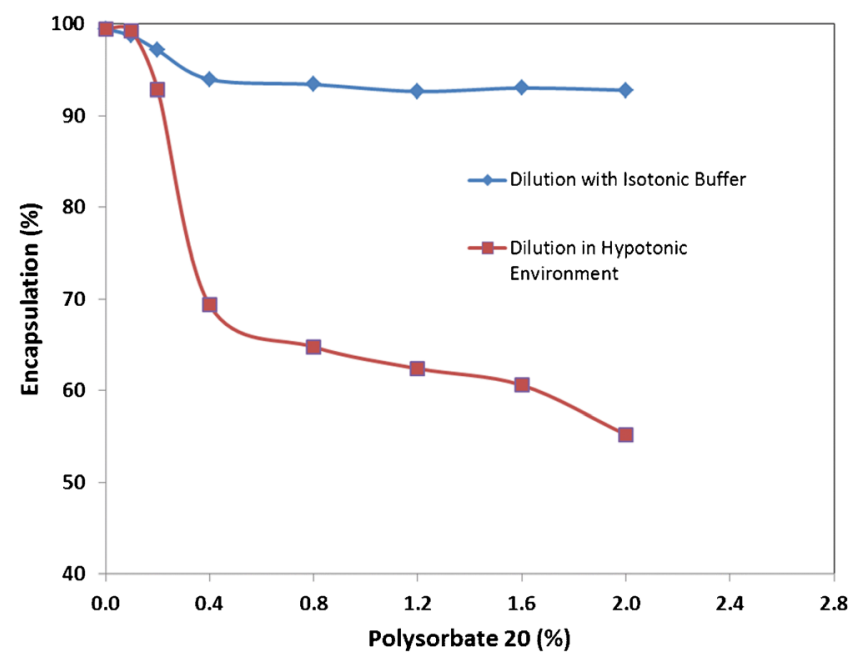

Fig. 1. The effect of addition of polysorbate 20 on the state of ciprofloxacin encapsulation when diluted with either water or isotonic histidine buffer, $\mathrm{pH} 6.0$ buffer. CFI at $50 \mathrm{mg} / \mathrm{ml}$ ciprofloxacin was diluted to a final concentration of $\sim 12.5 \mathrm{mg} / \mathrm{ml}$ with water or histidine buffer and then either an aliquot of 1 or $10 \%$ polysorbate 20 was added to achieve a final surfactant concentration of $0.1,0.2,0.4,0.8,1.2,1.6$, or $2.0 \%$. After vortexing and allowing each sample to equilibrate for at least $30 \mathrm{~min}$, the ciprofloxacin encapsulation state was determined by centrifugal filtration in duplicate. Adapted from reference (6) 


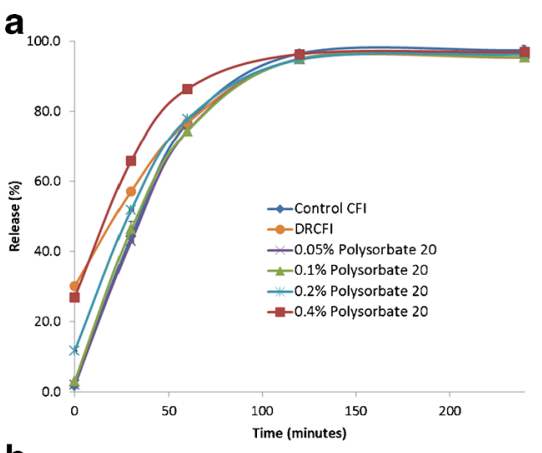

b

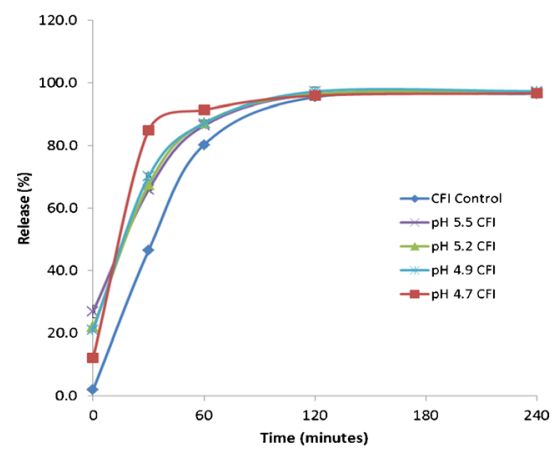

C

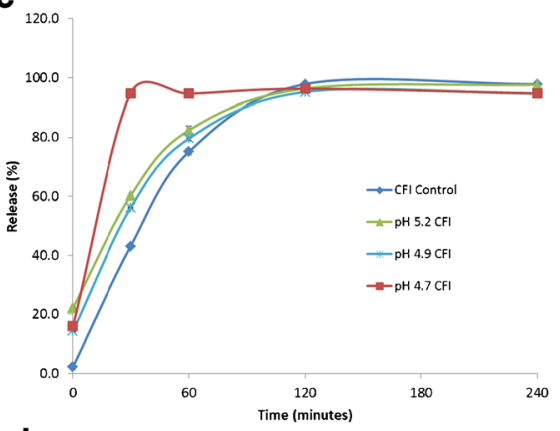

d

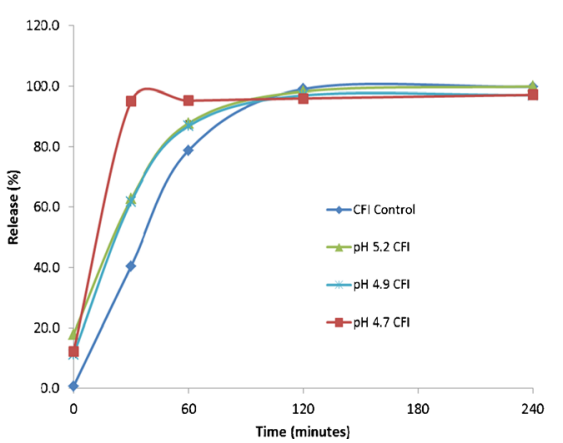

e

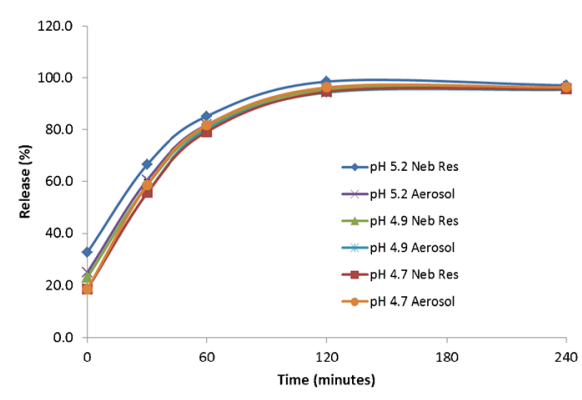

Fig. 2. Evaluation of the effect of liposomal composition on the IVR assay. The control CFI formulation (at $50 \mathrm{mg} / \mathrm{mL}$ ) and each of the $12.5 \mathrm{mg} /$ $\mathrm{mL}$ CFI formulations were diluted to $50 \mu \mathrm{g} / \mathrm{mL}$ CFI in HEPES buffered saline $(H B S)$ prior to a 1:1 dilution in bovine serum to measure the release of ciprofloxacin after incubation at $37^{\circ} \mathrm{C}$ for up to $4 \mathrm{~h}$. a IVR profiles for control CFI (blue diamonds), DRCFI (orange circles), CFI in $0.05 \%$ polysorbate 20 (purple crosses), CFI in $0.1 \%$ polysorbate 20 (green triangles), CFI in $0.2 \%$ polysorbate 20 (blue stars), and CFI in $0.4 \%$ polysorbate 20 (red squares). Fig. 2a reprinted with permission from reference 6. b IVR profiles at the initial time point for control CFI (blue diamonds), CFI in $0.4 \%$ polysorbate $20, \mathrm{pH} 5.5$ (purple crosses), CFI in $0.4 \%$ polysorbate 20 , pH 5.2 (green triangles), CFI in $0.4 \%$ polysorbate 20 , pH 4.9 (blue stars), and CFI in $0.4 \%$ polysorbate 20, $\mathrm{pH} 4.7$ (red squares). c IVR profiles at the 6-month time point for control CFI (blue diamonds), CFI in $0.4 \%$ polysorbate $20, \mathrm{pH} 5.2$ (green triangles), CFI in $0.4 \%$ polysorbate $20, \mathrm{pH} 4.9$ (blue stars), and CFI in $0.4 \%$ polysorbate $20, \mathrm{pH} 4.7$ (red squares). d IVR profiles at the 12-month time point for control CFI (blue diamonds), CFI in $0.4 \%$ polysorbate 20 , $\mathrm{pH} 5.2$ (green triangles), $12.5 \mathrm{mg} / \mathrm{ml} \mathrm{CFI} \mathrm{in} 0.4 \%$ polysorbate $20, \mathrm{pH} 4.9$ (blue stars), and $12.5 \mathrm{mg} / \mathrm{ml} \mathrm{CFI} \mathrm{in} 0.4 \%$ polysorbate 20 , pH 4.7 (red squares). e IVR profiles at the 6-month time point after nebulization for the nebulizer residual solution (Neb Res) and collected aerosol, respectively: CFI in $0.4 \%$ polysorbate $20, \mathrm{pH} 5.2$ (blue diamonds and purple crosses), CFI in $0.4 \%$ polysorbate $20, \mathrm{pH} 4.9$ (green triangles and blue stars), and CFI in $0.4 \%$ polysorbate $20, \mathrm{pH} 4.7$ (red squares and orange circles). Each value represents the mean $\pm \mathrm{SD}(n=3$ or 2$)$. The error bars which are not visible are smaller than the width of the data symbols

release of encapsulated drug occurs to a greater extent under hypotonic conditions (6). These encapsulation values remained fairly constant over the 2-year refrigerated storage period (Table III).

\section{IVR Profiles}

The IVR profiles of the three CFI formulations with adjusted $\mathrm{pH}$ values show similar characteristics to the IVR profile for the $\mathrm{pH} 5.5$ formulation without $\mathrm{pH}$ modification at the initial time point (Fig. 2b). The IVR "burst" values ranged between 12 and $27 \%$ free drug for the four formulations prior to incubation at $37^{\circ} \mathrm{C}$. The formulation with the lowest $\mathrm{pH}(\mathrm{pH} 4.7)$ contained the greatest encapsulated drug $(88 \%)$ and lowest free drug $(12 \%)$ while the unmodified formulation at the highest $\mathrm{pH}$ ( $\mathrm{pH}$ 5.5) had the lowest encapsulated drug (73\%) and highest free drug $(27 \%)$ in the IVR assay. These IVR "burst" values are comparable to the encapsulation values reported in Table III, as would be expected. The IVR profiles for the CFI formulations containing $0.4 \%$ polysorbate 20 at $\mathrm{pH} 4.9,5.2$, and 5.5 were

Table I. The Vesicle Size Distribution of the CFI Control and the pH Adjusted $12.5 \mathrm{mg} / \mathrm{ml} \mathrm{CFI} \mathrm{Formulations} \mathrm{Containing} 0.4 \%$ Polysorbate 20 After Refrigerated Storage for 2.5, 6, 12 and 24 Months. Vesicle Size Data are Reported as the Mean (in nm) and [SD]

\begin{tabular}{lcccc}
\hline Time (months) & CFI control & pH 5.2 CFI & pH 4.9 CFI & pH 4.7 CFI \\
\hline 0 & $90.5[25.7]$ & $90.6[17.6]$ & $91.8[25.8]$ & $93.4[19.1]$ \\
2.5 & $90.3[24.7]$ & $93.6[20.3]$ & $93.9[15.6]$ & $95.2[26.0]$ \\
6 & $90.8[20.5]$ & $93.6[21.1]$ & $94.1[24.0]$ & $96.5[27.0]$ \\
12 & $94.1[27.8]$ & $94.3[27.1]$ & $93.7[22.5]$ & $95.6[26.5]$ \\
24 & $88.7[22.0]$ & $98.8[36.2]$ & $99.9[27.9]$ & $97.0[26.3]$ \\
\hline
\end{tabular}

$12.5 \mathrm{mg} / \mathrm{mL}$ CFI Formulations in $0.4 \%$ polysorbate 20 at $\mathrm{pH} 5.2,4.9$, and 4.7 
Table II. The $\mathrm{pH}$ of the CFI Control and the $\mathrm{pH}$ Adjusted $12.5 \mathrm{mg} / \mathrm{ml}$ CFI Formulations Containing 0.4\% Polysorbate 20 After Refrigerated Storage for 1, 6, 12 and 24 Months

\begin{tabular}{lccc}
\hline Time (months) & $\mathrm{pH} \mathrm{5.2} \mathrm{CFI}$ & $\mathrm{pH} \mathrm{4.9} \mathrm{CFI}$ & $\mathrm{pH} \mathrm{4.7} \mathrm{CFI}$ \\
\hline 0 & 5.18 & 4.91 & 4.67 \\
1 & 5.14 & 4.89 & 4.63 \\
6 & 4.88 & 4.82 & 4.61 \\
12 & 5.05 & 4.93 & 4.71 \\
24 & 5.05 & 5.02 & 4.85 \\
\hline
\end{tabular}

$12.5 \mathrm{mg} / \mathrm{mL}$ CFI formulations in $0.4 \%$ polysorbate 20 at $\mathrm{pH} 5.2,4.9$, and 4.7

comparable to each other, but were significantly faster than for the control CFI formulation without $0.4 \%$ polysorbate 20 (Fig. 2b and Table IV). However, the pH 4.7 CFI formulation had an even faster IVR profile with most of its drug content released within the first $30 \mathrm{~min}$. At the end of the 4-h incubation period, all of the formulations reached a plateau representing $\sim 100 \%$ release of encapsulated drug.

The IVR profiles for the $\mathrm{pH} 5.2,4.9$, and 4.7 CFI formulations containing $0.4 \%$ polysorbate 20 were assayed after refrigerated storage for 6 months (Fig. 2c) and 1 year (Fig. 2d). The IVR profiles for the CFI formulations containing $0.4 \%$ polysorbate 20 at $\mathrm{pH} 5.2,4.9$, and 4.7 were relatively unchanged over this 1-year time frame with respect to their "burst" values, indicative of the amount of encapsulated drug, their rates of release (Table IV), and their plateau values, which represented $100 \%$ release of encapsulated drug.

\section{Aerosol Characterization}

Initially, CFI formulations containing $0.4 \%$ polysorbate 20 were evaluated using various jet nebulizers to characterize their aerosol performance. The presence of the surfactant resulted in excessive foaming and led to very low and inconsistent aerosol output rates thus rendering jet nebulization inappropriate for use with these formulations. A transition to a vibrating mesh nebulizer, the PARI eFlow rapid, was then made. The aerosol particle size distribution of the $12.5 \mathrm{mg} / \mathrm{ml}$ CFI preparation containing $0.4 \%$ polysorbate 20 , pH-5.5, was determined after mesh nebulization with a VMD of $3.74 \mu \mathrm{m}$ $(\mathrm{SD}=0.01 \mu \mathrm{m}, n=3)$ and a GSD of $1.72(\mathrm{SD}=0.02, n=3)$.

Table III. The Percent Encapsulation of Ciprofloxacin in the CFI Control and in the $\mathrm{pH}$ Adjusted $12.5 \mathrm{mg} / \mathrm{ml}$ CFI Formulations Containing $0.4 \%$ Polysorbate 20 After Refrigerated Storage for 1, 2.5, 6, 12, and 24 Months

\begin{tabular}{lcccc}
\hline $\begin{array}{l}\text { Time } \\
\text { (months) }\end{array}$ & $\begin{array}{l}\text { CFI } \\
\text { Control }\end{array}$ & $\begin{array}{l}\text { pH 5.2 } \\
\text { CFI }\end{array}$ & $\begin{array}{l}\text { pH 4.9 } \\
\text { CFI }\end{array}$ & $\begin{array}{l}\text { pH 4.7 } \\
\text { CFI }\end{array}$ \\
\hline 0 & 99.5 & 80.1 & 84.9 & 92.8 \\
1 & 98.9 & 77.0 & 84.7 & 91.1 \\
2.5 & 99.6 & 79.0 & 86.6 & 94.8 \\
6 & 99.1 & 76.5 & 81.2 & 89.6 \\
12 & 99.7 & 80.0 & 86.9 & 92.2 \\
24 & 99.3 & 80.8 & 85.4 & 92.0 \\
\hline
\end{tabular}

$12.5 \mathrm{mg} / \mathrm{mL}$ CFI Formulations in $0.4 \%$ polysorbate 20 at $\mathrm{pH} 5.2,4.9$, and 4.7
Aerosol Emitted Dose, and Effect on Vesicle Size and Encapsulation State

Using the PARI eFlow rapid, $5 \mathrm{~mL}$ of each formulation was nebulized ranging between 3.4 and 4.0 min, slightly faster than that for the CFI control without surfactant (Table V). The emitted dose (ED) values for the three experimental formulations ranged between 57 and $61 \%$ of the dose loaded in the nebulizer, lower than the $\sim 72 \%$ ED observed for the CFI Control (Table V). Given the shorter nebulization times, it is not surprising that the ED values would be lower for the experimental formulations. The lower ED values for the experimental formulations were likely a result of the small amount of foam observed in the reservoirs which may have reduced the formulation volume that was available to pass through the mesh. The mass balance recovery exceeded $90 \%$ on average. For all three experimental formulations, the mean vesicle size increased by $\sim 3 \mathrm{~nm}$ for the material recovered from the nebulizer reservoir and by an additional $\sim 30 \mathrm{~nm}$ for the collected aerosol (Table V). This is in contrast to the control CFI formulation for which there was no significant change in mean vesicle size for either the nebulizer reservoir or collected aerosol compartments (Table V). The encapsulation state of formulation recovered from the nebulizer reservoir decreased significantly for all three formulations to between 65 and $77 \%$ whereas the encapsulation state of the control CFI formulation was unchanged (Table V).

\section{One Month Analysis: Effect on Vesicle Size and Encapsulation State}

At the 1-month time point, the CFI formulations containing $0.4 \%$ polysorbate 20 at $\mathrm{pH} 5.2,4.9$, and 4.7 were aerosolized but this time, instead of collecting the aerosol in the BioSampler for subsequent analysis, aerosol droplets which coalesced inside the mouthpiece were evaluated for vesicle size and encapsulation state. This change was made in case the BioSampler collection process was contributing to changes in the state of the sample; e.g., the increase in vesicle size observed in the aerosol samples at the initial time point. For all three formulations, there was a small increase in the average vesicle size for the residual solution in the nebulizer reservoir, consistent with the initial assessment (Table VI). However, in contrast to the previous results at time zero, there was no additional increase in the vesicle size for the collected aerosol samples for all three formulations (Table VI). This result suggests that the BioSampler collection process was primarily responsible for the increase in the vesicle size upon aerosolization, not the mesh nebulization process itself. The encapsulation state for the formulation remaining in the nebulizer reservoir did not decrease as significantly for any of the three formulations as at the time zero analysis. However, the collected aerosol samples all decreased to an encapsulation state of between 70 and $80 \%$, consistent with what was observed at time zero using the BioSampler (Table V).

\section{Six Month Analysis: Effect on Vesicle Size, Encapsulation State and IVR Profile}

At the 6 month time point, the effect of aerosolization of the CFI formulations containing $0.4 \%$ polysorbate 20 at $\mathrm{pH} 5.2,4.9$, 
Table IV. The Normalized Rate of Release in the IVR Assay of the $\mathrm{pH}$ Adjusted $12.5 \mathrm{mg} / \mathrm{mL}$ CFI Formulations Containing $0.4 \%$ Polysorbate 20 After Refrigerated Storage for 1, 6, and 12 Months. The Initial Rate of Release in the IVR Assay is Defined as $100 \times\left(\mathrm{T}_{30}-\mathrm{T}_{0}\right) /\left(\mathrm{T}_{240}-\mathrm{T}_{0}\right)$ was Normalized by Dividing by the Initial Rate of Release of the CFI Control at the Same Time Point and Expressed as a Percentage

\begin{tabular}{|c|c|c|c|c|c|c|}
\hline \multirow[b]{3}{*}{ Formulation } & \multicolumn{3}{|c|}{ Normalized IVR (\%) } & \multicolumn{3}{|c|}{ Relative IVR (\% of control CFI) } \\
\hline & \multicolumn{3}{|c|}{ Time (months) } & \multicolumn{3}{|c|}{ Time (months) } \\
\hline & 1 & 6 & 12 & 1 & 6 & 12 \\
\hline Control CFI & 47.0 & 42.8 & 40.1 & 100 & 100 & 100 \\
\hline pH 5.5 CFI & 55.7 & ND & ND & 118 & ND & ND \\
\hline pH 5.2 CFI & 60.9 & 50.6 & 54.7 & 129 & 118 & 136 \\
\hline $\mathrm{pH} 4.9$ CFI & 64.3 & 52.2 & 58.9 & 137 & 122 & 147 \\
\hline $\mathrm{pH} 4.7 \mathrm{CFI}$ & 86.1 & 100.1 & 97.5 & 183 & 234 & 243 \\
\hline
\end{tabular}

$12.5 \mathrm{mg} / \mathrm{mL}$ CFI Formulations in $0.4 \%$ polysorbate 20 at pH 5.5, 5.2, 4.9, and 4.7. The normalized IVR equals $=100 \times\left(\mathrm{T}_{30 \mathrm{~min}}-\mathrm{T}_{0 \mathrm{~min}}\right) /\left(\mathrm{T}_{240 \mathrm{~min}}-\right.$ $\left.\mathrm{T}_{0 \min }\right)$. The relative $\mathrm{IVR}=100 \times\left(\right.$ normalized $\mathrm{IVR}_{\mathrm{SAMPLE}} /$ normalized $\left.\mathrm{IVR}_{\mathrm{CONTROL}}\right)$

$N D$ not done

and 4.7 was evaluated with respect to vesicle size, encapsulation state, and IVR profile. For all three formulations, the mean vesicle size increased by $2-3 \mathrm{~nm}$ for the sample recovered from the nebulizer reservoir and by a further 1-3 $\mathrm{nm}$ for the collected aerosol sample (Table VI), consistent with what was observed at the 1-month time point. The encapsulation state decreased slightly by 1 to $6 \%$ for all three formulations whether recovered from the nebulizer reservoir or after aerosolization (Table VI).

After 6 months stability, the $\mathrm{pH} 4.7,4.9$, and 5.2 CFI formulations were aerosolized and the collected aerosol and the residual formulation in the nebulizer reservoir were evaluated in the IVR assay. The IVR profiles of the collected aerosol samples were comparable to those for the residual formulation remaining in the nebulizer reservoir (Fig. 2e). The IVR profiles for the aerosolized $\mathrm{pH} 5.2$ and 4.9 CFI formulations containing $0.4 \%$ polysorbate 20 were also comparable to that for the unnebulized control samples at the 6month time point (Fig. 2c). However, for the $\mathrm{pH} 4.7 \mathrm{CFI}$ formulation containing $0.4 \%$ polysorbate 20 , the IVR profiles for both the collected aerosol and nebulizer reservoir samples (Fig. 2e) did not show as rapid a release as for the unnebulized control (Fig. 2c), but instead had IVR profiles that were similar to the CFI formulations at higher $\mathrm{pH}$.

\section{Cryo-TEM Imaging}

To examine if the physical structure of the liposomes was altered by nebulization, cryo-TEM imaging was performed. Cryo-TEM imaging of the control CFI without surfactant reveals a population of spherical, unilamellar vesicles between $\sim 50$ and $100 \mathrm{~nm}$ in diameter (Fig. 3a), consistent with that reported previously (15). Imaging was also performed on CFI diluted with $0.4 \%$ polysorbate 20 and water, to produce transient osmotic swelling, and an increase in free drug (Fig. 3b). The majority of these surfactant-associated liposomes looked qualitatively similar to that for control CFI (Fig. 3b vs. 3a); however, there was a minority of liposomes that were lighter, consistent with loss of encapsulated drug (6). Cryo-TEM imaging was not performed on the $\mathrm{pH}$ adjusted CFI formulations. After exposure of the $12.5 \mathrm{mg} / \mathrm{ml} \mathrm{CFI}$ formulation containing $0.4 \%$ polysorbate 20 at $\mathrm{pH} 5.5$ to the mesh nebulization process, the aerosol sample recovered in the mouthpiece was not compromised with respect to the liposome structure or integrity as evidenced by cryo-TEM analysis (Fig. 3c). There was no noticeable change in the size distribution of the liposomes or an increase in the proportion of liposomes with lighter shading, which would have suggested loss of encapsulated drug. There

Table V. Characterization of the Effect of Nebulization of the CFI Control and pH Adjusted $12.5 \mathrm{mg} / \mathrm{ml} \mathrm{CFI} \mathrm{Formulations} \mathrm{Containing} 0.4 \%$ Polysorbate 20. The Vesicle Size Data are Reported as the Mean (in nm) and [SD]. The State of Ciprofloxacin Encapsulation is Reported in Terms of Percentage. The Percent Recovery of Ciprofloxacin in the Nebulizer Reservoir and in the Collected Aerosol (emitted dose) Allows for a Calculation of the Mass Balance. The Nebulization Time is Recorded in Minutes

\begin{tabular}{|c|c|c|c|c|c|c|c|c|c|c|}
\hline \multirow[b]{2}{*}{ Formulation } & \multirow[b]{2}{*}{$\begin{array}{l}\text { Experiment } \\
\text { run no. }\end{array}$} & \multicolumn{3}{|c|}{ Mean vesicle size $(\mathrm{nm})[\mathrm{SD}]$} & \multicolumn{2}{|c|}{ Encapsulation (\%) } & \multicolumn{2}{|c|}{ Recovery (\%) } & \multirow[b]{2}{*}{$\begin{array}{l}\text { Mass } \\
\text { balance (\%) }\end{array}$} & \multirow[b]{2}{*}{$\begin{array}{l}\text { Neb. time } \\
(\mathrm{min})\end{array}$} \\
\hline & & $\begin{array}{l}\text { Before } \\
\text { Neb. }\end{array}$ & $\begin{array}{l}\text { Neb. } \\
\text { Res. }\end{array}$ & Aerosol & $\begin{array}{l}\text { Before } \\
\text { Neb. }\end{array}$ & $\begin{array}{l}\text { Neb. } \\
\text { Res. }\end{array}$ & $\begin{array}{l}\text { Neb. } \\
\text { Res. }\end{array}$ & Aerosol & & \\
\hline \multirow[t]{2}{*}{ CFI control } & $\# 1$ & 90.5 [25.7] & 91.6 [22.9] & 91.3 [22.7] & 99.5 & 98.9 & 21.6 & 73.2 & 94.8 & 5.0 \\
\hline & $\# 2$ & 90.5 [25.7] & 89.9 [22.3] & 90.8 [25.9] & 99.5 & 99.0 & 23.6 & 71.2 & 94.8 & 4.2 \\
\hline \multirow[t]{2}{*}{ pH 5.5 CFI } & $\# 1$ & $90.6[17.6]$ & 92.1 [24.9] & $127.9[77.6]$ & 70.2 & 67.6 & 34.9 & 58.2 & 93.1 & 3.5 \\
\hline & $\# 2$ & 90.6 [17.6] & $94.7[23.2]$ & $135.0[85.0]$ & 70.2 & 65.6 & 33.2 & 60.3 & 93.5 & 4.0 \\
\hline pH 5.2 CFI & ND & ND & ND & ND & 80.1 & ND & ND & ND & ND & ND \\
\hline \multirow[t]{2}{*}{$\mathrm{pH} 4.9 \mathrm{CFI}$} & $\# 1$ & 91.8 [25.4] & $94.8[28.1]$ & $128.7[76.6]$ & 84.9 & 70.5 & 30.5 & 61.0 & 91.5 & 3.7 \\
\hline & $\# 2$ & $91.8[25.4]$ & $94.6[29.0]$ & $125.3[74.5]$ & 84.9 & 73.4 & 33.8 & 57.2 & 91.0 & 3.4 \\
\hline \multirow[t]{2}{*}{ pH 4.7 CFI } & $\# 1$ & $93.4[19.1]$ & $96.2[26.0]$ & $125.9[73.0]$ & 92.8 & 77.0 & 30.6 & 59.1 & 89.8 & 3.9 \\
\hline & $\# 2$ & $93.4[19.1]$ & 96.3 [25.8] & $123.1[72.7]$ & 92.8 & 73.7 & 33.3 & 59.5 & 92.8 & 3.6 \\
\hline
\end{tabular}


Table VI. The Liposome Vesicle Size Distribution and the Percent Encapsulation of Ciprofloxacin After 1 and 6 Months Refrigerated Storage and Following Nebulization for the $\mathrm{pH}$ Adjusted $12.5 \mathrm{mg} / \mathrm{ml}$ CFI Formulations Containing $0.4 \%$ Polysorbate 20. Vesicle Size Data are Reported as the Mean (in nm) and [SD]. Duplicate Nebulization Experiments were Performed at the 1-Month Time Point Versus Single Experiments at the Six-Month Time Point

\begin{tabular}{|c|c|c|c|c|c|c|c|}
\hline \multirow[b]{2}{*}{ Formulation } & \multirow[b]{2}{*}{ Expt. run } & \multicolumn{3}{|c|}{ Mean vesicle size $(\mathrm{nm})[\mathrm{SD}]$} & \multicolumn{3}{|c|}{ Encapsulation (\%) } \\
\hline & & Before Neb. & Neb. Res. & Aerosol & Before Neb. & Neb. Res. & Aerosol \\
\hline \multicolumn{8}{|c|}{ 1-month analysis } \\
\hline \multirow[t]{2}{*}{ pH 5.2 CFI } & $\# 1$ & $91.1[19.7]$ & $90.8[24.2]$ & $92.6[32.0]$ & 76.6 & 76.6 & 74.8 \\
\hline & $\# 2$ & $91.1[19.7]$ & $95.2[16.7]$ & $95.8[32.2]$ & 76.6 & 77.0 & 73.1 \\
\hline \multirow[t]{2}{*}{ pH 4.9 CFI } & $\# 1$ & 91.8 [25.3] & $93.0[24.4]$ & 93.1 [33.7] & 85.2 & 82.8 & 73.4 \\
\hline & $\# 2$ & $91.8[25.3]$ & $94.1[26.9]$ & $94.7[29.7]$ & 85.2 & 82.4 & 79.0 \\
\hline \multirow[t]{2}{*}{$\mathrm{pH} 4.7 \mathrm{CFI}$} & $\# 1$ & $95.4[27.4]$ & $96.7[23.6]$ & 96.2 [27.9] & 92.6 & 89.8 & 71.7 \\
\hline & $\# 2$ & $95.4[27.4]$ & $99.3[27.5]$ & $99.7[30.8]$ & 92.6 & 89.1 & 81.2 \\
\hline \multicolumn{8}{|c|}{ 6-month analysis } \\
\hline \multicolumn{2}{|l|}{ pH 5.2 CFI } & $93.6[21.1]$ & $96.0[23.8]$ & $99.0[29.2]$ & 76.5 & 70.7 & 76.5 \\
\hline \multicolumn{2}{|l|}{ pH 4.9 CFI } & $94.1[24.0]$ & $96.5[24.7]$ & $97.6[29.8]$ & 81.2 & 75.7 & 77.2 \\
\hline \multicolumn{2}{|l|}{ pH 4.7 CFI } & $96.5[27.0]$ & 98.6 [23.1] & $101.2[34.2]$ & 89.6 & 85.4 & 84.9 \\
\hline
\end{tabular}

was also no evidence of deformed liposomes or liposomal debris, which might be indicative of vesicle rupture during the mesh nebulization process.

\section{DISCUSSION}

We have investigated the interaction of polysorbate 20 with a liposomal ciprofloxacin formulation with the goal to develop novel formulations with modified encapsulation states and release properties which could then be used in nebulizers. Our strategy was to mix polysorbate 20 with the liposomes, in conjunction with osmotic swelling, so that the liposome vesicles retain their integrity, but have altered drug release rates (6). The amount of unencapsulated ciprofloxacin increased with greater concentrations of surfactant (Fig. 1) and above $0.4 \%$ polysorbate 20 there was a more rapid release profile in the IVR assay (Fig. 2a). The encapsulation states of the CFI formulations containing polysorbate 20 were maintained on long-term stability (Table III) which supports the model that sublytic concentrations of surfactant form transient pores in liposome bilayers allowing release of some of the encapsulated drug before the membrane barrier is fully recovered $(20,21)$. These liposomes might then be expected to maintain their structural integrity for prolonged time periods as was observed in our 2year stability study. The cryo-TEM micrographs of mixtures of CFI and low levels of polysorbate 20 are practically indistinguishable from CFI alone (Figs. 3a, b). The micrographs show predominantly spherical, unilamellar liposomes between around 50 and $100 \mathrm{~nm}$ for the control CFI liposomes, as well as for the surfactant-associated liposomes prior to and after mesh nebulization (Fig. 3c).

Because ciprofloxacin is poorly soluble at neutral $\mathrm{pH}$, the external $\mathrm{pH}$ of the surfactant-liposome mixture was reduced to 5.2, 4.9, and 4.7 to improve the likelihood that the released ciprofloxacin would remain in solution during long-term refrigerated storage. The $\mathrm{pH} 4.7$ and 4.9 preparations retained good appearance after 2-year refrigerated storage in contrast to the $\mathrm{pH} 5.5$ preparation which formed ciprofloxacin crystals within $48 \mathrm{~h}$. The appearance of the $\mathrm{pH} 5.2$ preparation showed some small particulates but otherwise showed no further deterioration after 24 months refrigerated storage. The intentional reduction in $\mathrm{pH}$ in these $\mathrm{CFI}$ formulations was associated with an increase in encapsulation relative to the $\mathrm{pH} 5.5$ formulation, with the greatest encapsulation for the lowest $\mathrm{pH}$ CFI formulation (Table III). Because acetate buffer was used to lower the $\mathrm{pH}$ for the $\mathrm{pH}$ modified formulations, in place of some of the dilution water, the external environment would be less hypotonic. This observation may explain the higher levels of drug encapsulation for the lower $\mathrm{pH}$ formulations. Notably however, once this new encapsulation state was established, it did not fluctuate during the course of the 2-year refrigerated storage (Table III). Use of acetic acid, instead of acetate buffer, to perform the dilutions could be used in the future to reduce the $\mathrm{pH}$ while maintaining a more hypotonic environment so that the encapsulation state could be reduced further, similar to the levels for the $\mathrm{pH}$ 5.5 CFI formulation.

There was also a slight increase in vesicle size with reduction in $\mathrm{pH}$ upon preparation but there were no further vesicle size changes throughout the course of the 2-year refrigerated storage (Table II). The IVR profiles for the liposome-surfactant preparations at $\mathrm{pH} 5.2$ and 4.9 were similar to that for the $\mathrm{pH} 5.5$ preparation (Fig. 2b), and retained that modified release profile through 12 months refrigerated storage (Fig. 2c, d). In contrast, the $\mathrm{pH} 4.7$ surfactant-liposome preparation had a much faster release profile in the IVR assay (Fig. 2b) that remained unchanged after 1-year refrigerated storage (Fig. 2c, d). It is unclear why the lowest $\mathrm{pH}$ liposomesurfactant preparation had an even faster release rate but it may be related to changes in the transmembrane $\mathrm{pH}$ gradient which is used to load the drug during manufacture of the liposomes $(17,18)$.

Many liposomal products have been developed with the intention for inhalation administration to treat lung disease (2). However, very few have been found to be robust to the nebulization process (2). Liposomes containing surfactant have historically been even less stable than traditional liposomes in response to nebulization with changes in vesicle size or encapsulation $(14,22)$. Ultradeformable liposome formulations containing salbutamol sulfate and comprised of soy phosphatidylcholine (PC), polysorbate 80, and cholesterol 

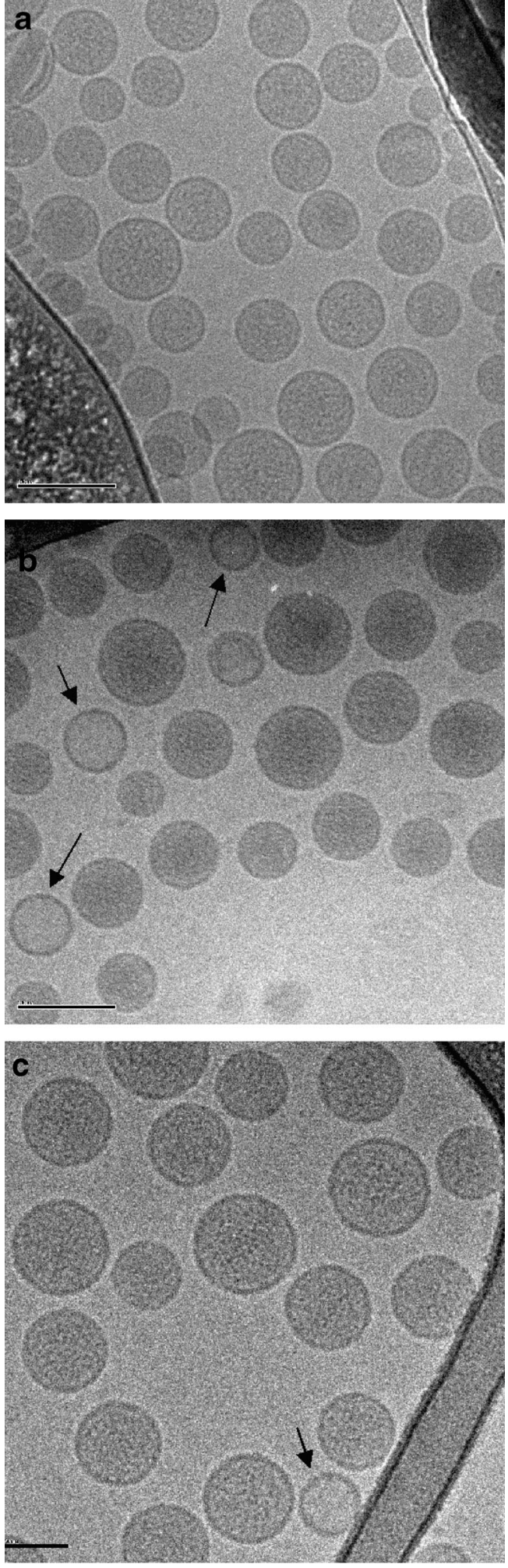

Fig. 3. Cryo-TEM micrographs of various preparations of liposomal ciprofloxacin. The scale bar in the bottom left-hand corner of each micrograph is $100 \mathrm{~nm}$ for $\mathbf{a}, \mathbf{b}$, and c. All samples were applied at a concentration of $\sim 10 \mathrm{mg} / \mathrm{mL}$ liposomes. a $12.5 \mathrm{mg} / \mathrm{ml} \mathrm{CFI}$; b $12.5 \mathrm{mg} /$ $\mathrm{ml} \mathrm{CFI} \mathrm{in} 0.4 \%$ polysorbate $20, \mathrm{pH} 5.5$; and $\mathbf{c} 12.5 \mathrm{mg} / \mathrm{ml}$ CFI in $0.4 \%$ polysorbate $20, \mathrm{pH} 5.5$, after mesh nebulization

were evaluated in jet, ultrasonic, and mesh nebulizers and all combinations were found to have marked losses in drug encapsulation and changes in vesicle size (14). Liposomes containing cyclosporine and comprised of soy $\mathrm{PC}$ and polysorbate 80 were exposed to mesh nebulization and the average vesicle size doubled from $51 \mathrm{~nm}$ before nebulization to $107 \mathrm{~nm}$ after nebulization (22). However, there was no reported loss in drug encapsulation, presumably because the poorly soluble cyclosporine drug remained associated with the hydrophobic liposome bilayers (23). Specially designed liposomal ciprofloxacin formulations have already been shown to be stable to jet nebulization with no meaningful changes in vesicle size or loss in drug encapsulation $(19,24)$. After mesh nebulization, surfactant-associated liposomal ciprofloxacin formulations (containing $0.4 \%$ polysorbate 20 ) showed a small increase in mean vesicle size (by a few $\mathrm{nm}$ ) and the drug encapsulation also decreased to $70-85 \%$ (Table VI). However, the degree of modification to these surfactant-associated liposomal ciprofloxacin formulations is less pronounced than for those liposomal formulations discussed above $(14,22)$.

Liposomal ciprofloxacin formulations containing $0.4 \%$ polysorbate 20 possessed modified release properties (Fig. 2a), yet were found to retain those IVR characteristics after 12 months refrigerated storage (Fig. 2d). In addition, after 6 months storage followed by mesh nebulization, all formulations demonstrated relatively comparable encapsulation states and IVR profiles (Fig. 2e). One unexplained finding related to this observation is why the $\mathrm{pH} 4.7 \mathrm{CFI}$ preparation, with the most rapid IVR profile prior to nebulization (Fig. 2c), has an IVR profile comparable to those for the $\mathrm{pH} 4.9$ and 5.2 preparations after mesh nebulization (Fig. 2e). While no explanation is evident, this result suggests that the mesh nebulization process has an effect on the low $\mathrm{pH}$ CFI formulation, even if that effect was only to make its IVR profile similar to that of the higher $\mathrm{pH}$ CFI formulations. Another interesting observation is that this change in IVR profile occurred not only for the collected aerosol sample which passed through the mesh, but also for the residual formulation remaining in the reservoir, upstream of the mesh. This phenomenon of alteration to a liposomal formulation upstream of the mesh (in addition to modification to the formulation which passed through the mesh), due to the energetics of the vibrating mesh, has been reported previously (2). Similar to our data for the $\mathrm{pH}=4.7$ formulation, liposomal formulations of salbutamol sulfate showed changes in vesicle size $(14)$ and encapsulation $(14,25)$ for the residual formulations in the nebulizer reservoirs.

\section{CONCLUSIONS}

We have demonstrated that the encapsulation state and IVR properties of a liposomal ciprofloxacin formulation, $12.5 \mathrm{mg} / \mathrm{ml} \mathrm{CFI} \mathrm{containing} 0.4 \%$ polysorbate 20 , could be modified post manufacture by the addition of surfactant while still retaining acceptable long-term stability when formulated between a $\mathrm{pH}$ of 4.7 and 5.2. These modified formulations were nebulized using a vibrating mesh device to produce aerosols in the respirable size range, appropriate for delivery to the airways to treat lung disease. While there were small changes in the encapsulation state and vesicle size following mesh nebulization, these formulations retained a faster IVR profile than for the unmodified CFI control formulation. Future research efforts will focus on how the changes observed in 
the in vitro release rate translate in the in vivo setting. It may be possible one day to personalize inhaled therapy of liposomal formulations by selecting a surfactant, at a specified concentration, $\mathrm{pH}$, and osmolarity, to achieve a specific, desired change in encapsulation state and/or IVR properties for a specific individual.

\section{ACKNOWLEDGMENTS}

The authors acknowledge the facilities, and the scientific and technical assistance of the Australian Microscopy \& Microanalysis Research Facility at the Electron Microscope Unit, The University of New South Wales, and in particular, Judy Loo Ching Yee and Delfine Cheng who provided assistance with the cryo-TEM analysis of the samples. The authors thank Paul Young and Daniela Traini of the Woolcock Institute of Medical Research, and Francis Dayton, Sujata Mudumba, and Jim Blanchard of Aradigm Corp. for fruitful discussions.

\section{REFERENCES}

1. Drummond DC, Noble CO, Hayes ME, Park JW, Kirpotin DB. Pharmacokinetics and in vivo drug release rates in liposomal nanocarrier development. J Pharm Sci. 2008;97:4696-740.

2. Cipolla D, Gonda I, Chan HK. Liposomal formulations for inhalation. Ther Deliv. 2013;4(8):1047-72.

3. Cipolla D, Redelmeier T, Eastman S, Bruinenberg P, Gonda I. Liposomes, niosomes and proniosomes-a critical update of their (commercial) development as inhaled products. In: Dalby RN, Byron PR, Peart J, Suman JD, Farr SJ, Young PM, editors. Respiratory drug delivery Europe 2011. River Grove: Davis Healthcare Int'l Publishing; 2011. p. 41-54.

4. Sharma A, Sharma US. Liposomes in drug delivery: progress and limitations. Int J Pharm. 1997;154(2):123-40.

5. Bonacucina G, Cespi M, Misici-Falzi M, Palmieri GF. Colloidal soft matter as drug delivery system. J Pharm Sci. 2009;98(1):1-42.

6. Cipolla D, Wu H, Eastman S, Redelmeier T, Gonda I, Chan H-K. Modifying the release properties of liposomes toward personalized medicine. J Pharm Sci. 2014;103(6):1851-62.

7. Taylor KM, Newton JM. Liposomes for controlled delivery of drugs to the lung. Thorax. 1992;47(4):257-9.

8. Schreier H, Gonzalez-Rothi RJ, Stecenko AA. Pulmonary delivery of liposomes. J Control Rel. 1993;47(4):257-9.

9. Monforte V, Lopez-Sanchez A, Zurbano F, Ussetti P, Sole A, Casals C, et al. Prophylaxis with nebulized liposomal amphotericin B for Aspergillus infection in lung transplant patients does not cause changes in the lipid content of pulmonary surfactant. J Heart Lung Transplant. 2013;32(3):313-9.

10. Cipolla D, Chan H-K. Inhaled antibiotics to treat lung infection. Pharm Patent Analyst. 2013;2(5):647-63.

11. Clancy JP, Dupont L, Konstan MW, et al. Phase II studies of nebulised Arikace in CF patients with Pseudomonas aeruginosa infection. Thorax. 2013;68(9):818-25.
12. Bilton D, Pressler T, Fajac I, et al. Phase 3 efficacy and safety data from randomized, multicenter study of liposomal amikacin for inhalation (ARIKACE) Compared with TOBI in cystic fibrosis patients with chronic infection due to Pseudomonas Aeruginosa. Pediatr Pulmonol. 2013;48(S36):207-453.

13. Serisier DJ, Bilton D, De Soyza A, et al. Inhaled, dual release liposomal ciprofloxacin in non-cystic fibrosis bronchiectasis (ORBIT-2): a randomised, double-blind, placebo-controlled trial. Thorax. 2013;68:812-7.

14. Elhissi AM, Giebultowicz J, Stec AA, Wroczynski P, Ahmed W, Alhnan MA, et al. Nebulization of ultradeformable liposomes: the influence of aerosolization mechanism and formulation excipients. Int J Pharm. 2012;436(1-2):519-26.

15. Ong HX, Traini D, Cipolla D, Gonda I, Bebawy M, Agus H, et al. Liposomal nanoparticles control the uptake of ciprofloxacin across respiratory epithelia. Pharm Res. 2012;29(12):3335-46.

16. Cipolla D, Wu H, Eastman S, Redelmeier T, Gonda I, Chan H-K. Development and characterization of an in vitro release assay for liposomal ciprofloxacin for inhalation. J Pharm Sci. 2013. doi:10.1002/jps.23795.

17. Yim D, Blanchard JD, Mudumba S, Eastman S, Manda K, Redelmeier T, et al. The development of inhaled liposome-encapsulated ciprofloxacin to treat cystic fibrosis. In: Dalby RN, Byron PR, Peart J, Suman JD, Farr SJ, editors. Respiratory drug delivery 2006. River Grove: Davis Healthcare Int'l Publishing; 2006. p. 425-8.

18. Webb MS, Boman NL, Wiseman DJ, Saxon D, Sutton K, Wong $\mathrm{KF}$, et al. Antibacterial efficacy against an in vivo Salmonella typhimurium infection model and pharmacokinetics of a liposomal ciprofloxacin formulation. Antimicrob Agents Chemother. 1998;42(1):45-52.

19. Cipolla D, Dayton F, Fulzele S, Gabatan E, Mudumba S, Yim D, et al. Inhaled liposomal ciprofloxacin: in vitro properties and aerosol performance. In: Dalby RN, Byron PR, Peart J, Suman JD, Farr SJ, Young PM, editors. Respiratory drug delivery 2010. River Grove: Davis Healthcare Int'l Publishing; 2010. p. 409-14.

20. Ruiz J, Goni FM, Alonso A. Surfactant-induced release of liposomal contents. A Surv of Methods and Results Biochim Biophys Acta. 1988;937(1):127-34.

21. Memoli A, Annesini MC, Petralito S. Surfactant-induced leakage from liposomes: a comparison among different lecithin vesicles. Int J Pharm. 1999;184(2):227-35.

22. Egle R, Bitterle E, Gruber F, Keller M. Characterization of a liposomal ciclosporin A formulation before and after lyophilization and nebulization. AAPS National Meeting, 2008. AM-0800983.

23. Behr J, Zimmermann G, Baumgartner R, et al. Lung deposition of a liposomal cyclosporine A inhalation solution in patients after lung transplantation. J Aerosol Med Pulm Drug Deliv. 2009;22(2):121-30.

24. Cipolla D, Wu H, Chan J, Chan H-K, Gonda I. Liposomal ciprofloxacin for inhalation retains integrity following nebulization. In: Dalby RN, Byron PR, Peart J, Suman JD, Farr SJ, Young PM, editors. Respiratory drug delivery Europe 2013. River Grove: Davis Healthcare Int'l Publishing; 2013. p. $237-42$.

25. Elhissi AM, Faizi M, Naji WF, Gill HS, Taylor KM. Physical stability and aerosol properties of liposomes delivered using an air-jet nebulizer and a novel micropump device with large mesh apertures. Int J Pharm. 2007;334(1-2):62-70. 